\title{
NUMERICAL INTEGRATION OF THE AXISYMMETRIC ROBINSON-TRAUTMAN EQUATION BY A SPECTRAL METHOD
}

\author{
D. A. PRAGER' and A. W.-C. LUN'
}

(Received 16 January 1995; revised 1 April 1999)

\begin{abstract}
We have adapted the Spectral Transform Method, a technique commonly used in non-linear meteorological problems, to the numerical integration of the Robinson-Trautman equation. This approach eliminates difficulties due to the $S^{2} \times \mathbb{R}^{+}$topology of the equation. The method is highly accurate for smooth data and is numerically robust. Under spectral decomposition the long-time equilibrium state takes a particularly simple form: all nonlinear $(l \geq 2)$ modes tend to zero. We discuss the interaction and eventual decay of these higher order modes, as well as the evolution of the Bondi mass and other derived quantities. A qualitative comparison between the Spectral Transform Method and two finite difference schemes is given.
\end{abstract}

\section{Introduction}

The Robinson-Trautman [5] (RT) metric describes a class of vacuum spacetimes whose evolution are governed by a single field equation. Physically an RT spacetime describes a distorted non-rotating black hole which radiates energy in the form of purely outgoing gravitational waves, settling down to the Schwarzschild solution in the limit of large retarded time.

For all but the most trivial initial conditions exact solutions to the RT equation are unavailable. Nevertheless, several strong results concerning the global behaviour of the RT equation have been proved: Chruściel [2] has shown that solutions exist for quite general initial conditions; Lukács et al. [3] established uniqueness; in the limit of large retarded time the RT spacetime settles down to the Schwarzschild solution [3]; a number of conserved and monotonically decreasing (Lyapunov) quantities are known $[3,6]$.

Of these quantities the Bondi mass is the most readily interpretable: it gives a

\footnotetext{
'Department of Mathematics, Monash University, Clayton, Victoria 3168, Australia.

(C) Australian Mathematical Society 1999, Serial-fee code 0334-2700/99
} 
measure of the total mass-energy on a null-slice. Accordingly, we expect it to be a Lyapunov functional and strictly positive on physical grounds.

Our approach to the problem of numerical integration works primarily in a spectral representation, which describes the evolution of the RT equation in terms of a sum of spherical harmonics [1]. Spatial derivatives are evaluated in this spectral basis, where they are particularly simple. Non-linear operations take place in a pointwise basis, so it is necessary to transform back and forth between the two bases. The matrices which arise in this approach are almost full, making computations more intensive than in finite difference methods (which work exclusively in the pointwise basis and give rise to banded matrices) for problems of similar spatial resolution. However, the greater accuracy and convenience (from an analytic viewpoint) of the spectral transform method offsets this limitation making it the method of choice.

We also mention a more radical finite difference scheme, based on an (arbitrarily fine) triangulation of the sphere [4], which is applicable to the non-axisymmetric RT equation. We expect that this scheme will be superseded by a Spectral Transform approach.

\section{The axisymmetric Robinson-Trautman equation}

The RT equation describes a quantity $f$ defined on the two-sphere $S^{2}$ which settles down to a superposition of the $l=0$ and $l=1$ spherical harmonics in the limit of large $u$ (retarded time). $f$ may be interpreted as a conformal factor which describes a topological two-sphere (for fixed $u$ ).

The assumption of axisymmetry ( $\phi$ independence) reduces the 2 (space) +1 (retarded time) dimensional RT equation to the $1+1$ dimensional axisymmetric RobinsonTrautman (ART) equation. The use of the $z:=\cos \theta$ coordinate eliminates the troublesome coordinate singularities at the poles of the two-sphere, induced by the use of spherical-polar coordinates. The ART equation is given by

$$
\begin{gathered}
f_{{ }_{u}}=-f^{4}\left(\Delta_{0} \Delta_{0}+2 \Delta_{0}\right) f+f^{3}\left\{\left(1-z^{2}\right) \partial_{z z} f\right\}^{2}, \\
f=f(u, z): \mathbb{B}^{+} \times[-1,1] \rightarrow \mathbb{R}, \\
I C: f(0, \cdot)=f_{0}(\cdot), \quad f_{0}:[-1,1] \rightarrow \mathbb{R} .
\end{gathered}
$$

Here $\Delta_{0} \equiv\left(1-z^{2}\right) \partial_{z z}-2 z \partial_{z}+\left(1-z^{2}\right)^{-1} \partial_{\phi \phi}$ is the Laplacian operation on $S^{2}$ and $f_{0}$ is taken to be continuous.

Two important global quantities are the surface area of the topological two-sphere (which is conserved and set to $4 \pi$ by convention) and the Bondi mass (one of two 
Lyapunov quantities - see Singleton [6]):

$$
\begin{aligned}
4 \pi & =2 \pi \int_{-1}^{1} \frac{1}{f(u, z)^{2}} d z, \\
M_{b}(u) & =\frac{1}{2} \int_{-1}^{1} \frac{1}{f(u, z)^{3}} d z .
\end{aligned}
$$

The Bondi mass decreases as energy is carried to null-infinity by gravitational waves.

Our numerical schemes do not conserve surface area exactly and do not guarantee the monotonicity of the Bondi mass. However, the computational analogues of these quantities are extremely useful in testing the accuracy of simulations.

\section{The Spectral Transform Method}

3.1. Time-stepping As is usually the case for parabolic equations, explicit timestepping schemes are unstable except for unreasonably small time-steps. Instead we choose to approximate the ART equation at time-step $k+1 / 2$ by

$$
\frac{f^{[k+1]}-f^{[k]}}{\Delta u}=\left[\tilde{f}^{4} \mathscr{L}+\tilde{f}^{3} \mathscr{G} \tilde{f} \mathscr{G}\right] \frac{f^{[k+1]}+f^{[k]}}{2}:=\mathscr{D}(\tilde{f}) \frac{f^{[k+1]}+f^{[k]}}{2}
$$

in the style of Crank and Nicolson. The terms $\mathscr{L}$ and $\mathscr{G}$ are finite-dimensional approximations (either of spectral or finite difference type) to $-\left(\Delta_{0} \Delta_{0}+2 \Delta_{0}\right)$ and $\left(1-z^{2}\right) \partial_{z z}$ respectively. The symbol $\tilde{f}$ is short-hand for $f^{[k+1 / 2]}$.

Rearranging, we obtain a 'linear' equation for $f^{[k+1]}$ :

$$
\left[\mathscr{D}(\tilde{f})-\frac{2}{\Delta u} \mathscr{I}\right] f^{[k+1]}=-\left[\mathscr{D}(\tilde{f})-\frac{2}{\Delta u} \mathscr{I}\right] f^{[k]} .
$$

The idea of non-linear Crank-Nicolson is to iterate this equation with successively better approximations to $\tilde{f}$ and $f^{[k+1]}$. We represent an evolution step (solution of (4) with specified $\Delta u, \tilde{f}$ and $f^{[k]}$ ) by the operation

$$
f^{[k+1]} \leftarrow E\left(\Delta u, \tilde{f}, f^{[k]}\right)=f^{[k+1]} .
$$

Implementing $E$ involves computing the linearized operator $\mathscr{D}(\tilde{f})$ and solving the system of linear equations.

A simple predictor-corrector (PC) scheme can be constructed as follows:

Predictor: $\tilde{f} \leftarrow E\left(\frac{\Delta u}{2}, f^{[k]}, f^{[k]}\right)$,

Corrector: $f^{[k+1]} \leftarrow E\left(\Delta u, \tilde{f}, f^{[k]}\right)$. 
This type of scheme (just one Corrector step) was implemented by Singleton [6]. He used a finite difference scheme in the $\theta$ coordinate. This led to complications at the poles. By using the coordinate $z=\cos \theta$, these difficulties are avoided.

Rather than accept the result of a single PC step, we allow $M$ corrector steps:

Predictor: $\tilde{f} \leftarrow E\left(\frac{\Delta u}{2}, f^{[k]}, f^{[k]}\right)$,

Corrector (1): $f_{1}^{*} \leftarrow E\left(\Delta u, \tilde{f}, f^{[k]}\right)$,

Corrector $(m): f_{m}^{*} \leftarrow E\left(\Delta u, \frac{f^{[k]}+f_{m-1}^{*}}{2}, f^{[k]}\right), \quad m=2 ; \ldots, M$,

New f: $f^{[k+1]} \leftarrow f_{M}^{*}$.

If the system fails to converge after a few iterations then the choice of $\Delta u$ is probably too large. We obtained excellent results by fixing $M=2$ and using a simple (non-extrapolating) adaptive time-stepping scheme to adjust $\Delta u$.

3.2. Calculation of spatial derivatives If we write $f(u, z)$ as a superposition of Legendre polynomials,

$$
f(u, z)=q_{0}(u) P_{0}(z)+q_{1}(u) P_{1}(z)+\cdots+q_{n-1}(u) P_{n-1}(z),
$$

we find that $\Delta_{0} P_{l}(z)=-l(l+1) P_{l}(z)$ and consequently $\mathscr{L} P_{l}(z) \rightarrow-(l-1) l(l+$ 1) $(l+2) P_{l}(z)$, so in the spectral basis, where $f \sim q=\left[q_{0}, q_{1}, \ldots, q_{(n-1)}\right], \mathscr{L}:=$ diagonal $[0,0,-24,-120, \ldots]$. The derivative operation $\mathscr{G}$ is given by the uppertriangular matrix

$$
\left(\begin{array}{cccccccc}
0 & 0 & 2 & 0 & 2 & 0 & 2 & \ldots \\
\cdot & 0 & 0 & 6 & 0 & 6 & 0 & \ldots \\
& & -2 & 0 & 10 & 0 & 10 & \ldots \\
& & & -6 & 0 & 14 & 0 & \ldots \\
& & & & -12 & 0 & 18 & \ldots \\
& & & & -20 & 0 & \ldots \\
& & & & & & -30 & \ldots \\
& & & & & & & \ddots
\end{array}\right)
$$

In contrast to the simplicity of the derivative operations $L$ and $G$, the non-linear operations of vector-vector $\left(e . g \cdot \tilde{f}^{2}\right)$ and vector-matrix $(e . g . \tilde{f} \mathscr{G})$ multiplication appear extremely messy in the spectral basis. The optimal approach is to transform to a pointwise basis in order to perform these operations and then transform back to the spectral basis.

If the nodes in the pointwise basis are chosen to be the zeroes of the $n$th Legendre polynomial then the spectral to pointwise transformation $\mathscr{P}$ and its inverse $\mathscr{P}^{-1}$ (which can be written down explicitly) are well-conditioned. Note that $f=\mathscr{P} q$.

Given two vectors $a$ and $b$ defined pointwise, the vector-vector product $a b$ is obtained by multiplying components pointwise, for example $(a b)_{i}:=a_{i} b_{i}$. 


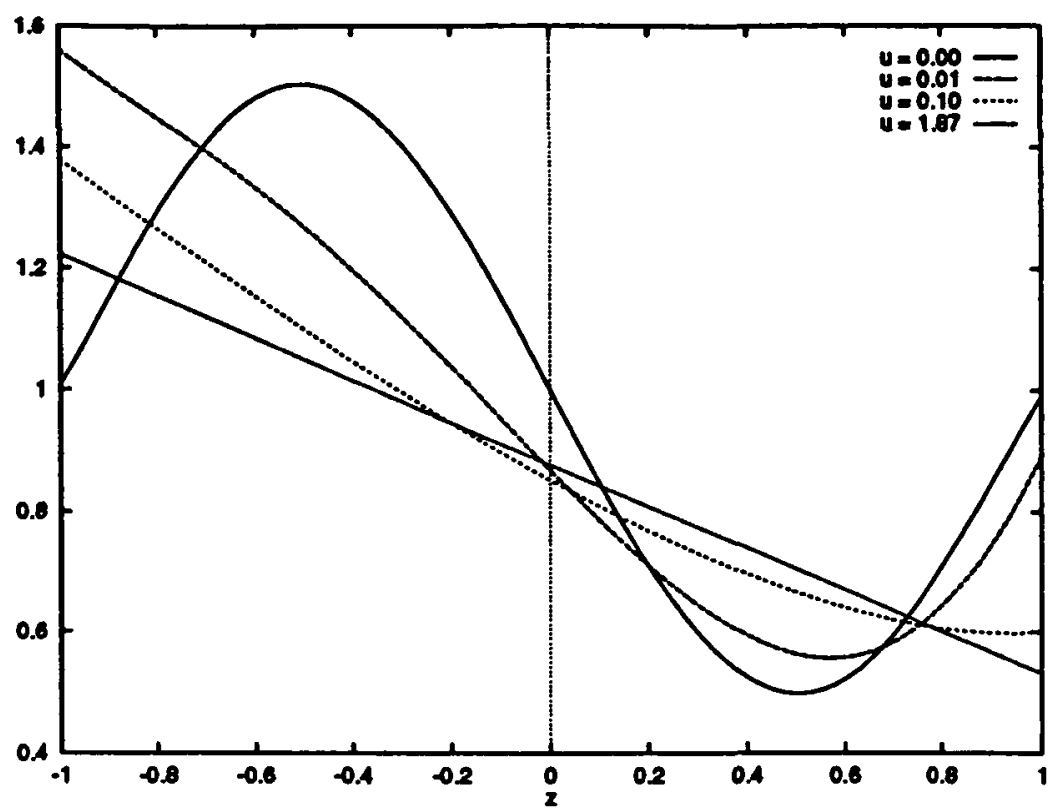

FIGURE 1. Snapshots of the evolution

To obtain a consistent definition of vector-matrix multiplication we can invoke associativity, which requires that $(a M) b=a(M b)$. Hence $(a M)_{i j}:=a_{i} M_{i j}$.

The spatial part of the RT equation in the spectral representation is

$$
\mathscr{D}(\tilde{q}):=\mathscr{P}^{-1}\left[(\mathscr{P} \tilde{q})^{4} \mathscr{P} \mathscr{L}+(\mathscr{P} \tilde{q})^{3} \mathscr{P} \mathscr{G} \tilde{q} \mathscr{P} \mathscr{G}\right] .
$$

Substituting $q$ for $f$ in (4), we can evolve forward in retarded time.

\section{A numerical example}

We consider an illustrative example: initial conditions specified by $f_{0}(z)=1-$ $0.5 \sin \pi z$, so all even modes other than $l=0$ are initially zero.

A spatial resolution of 21 modes was used - higher resolutions only cause minute changes to the results. For other initial conditions a higher spatial resolution may be necessary.

No effort was made to scale the initial surface area to the traditional value of $4 \pi$. In order to perform such a rescaling it would be necessary to multiply $f_{0}$ by a constant, which would be equivalent to a simple rescaling of both $f$ and $u$.

Figure 1 shows a sequence of snapshots of $f$ at fixed values of $u$, as $f$ evolves from a sinusoid into a straight line, representing the steady-state. Although this is a 


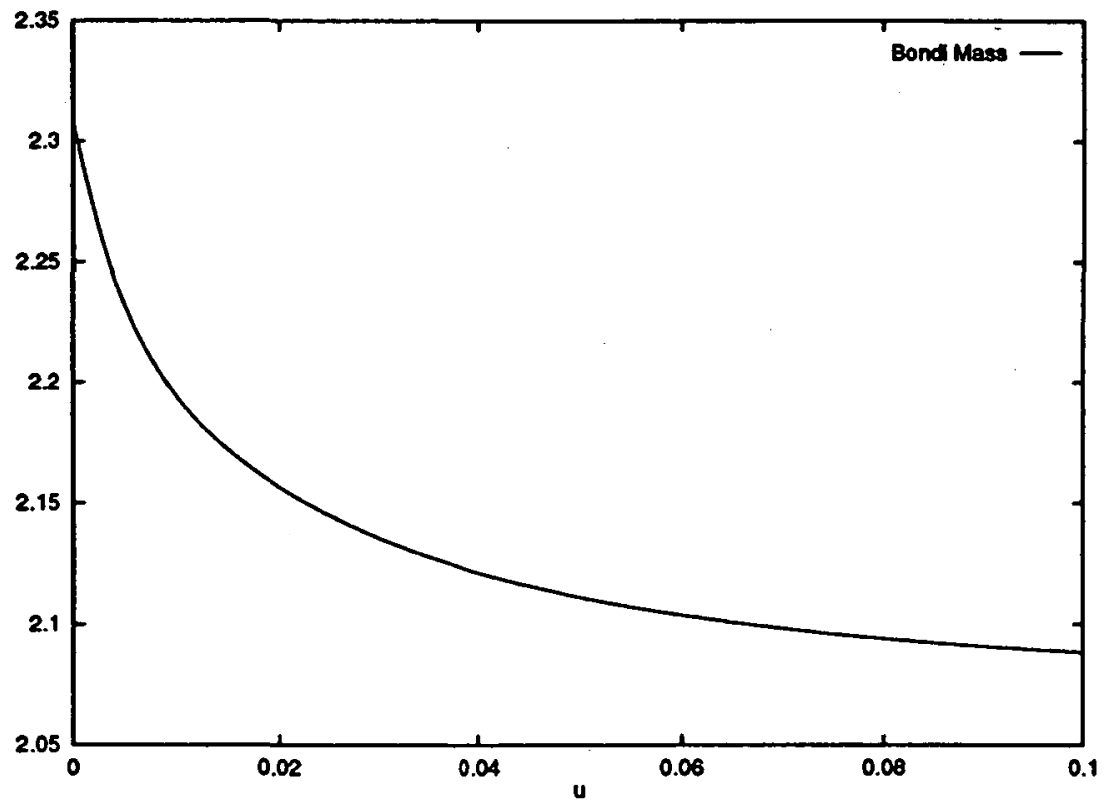

FIGURE 2. Monotonic decay of the Bondi mass (tends to 2.08)

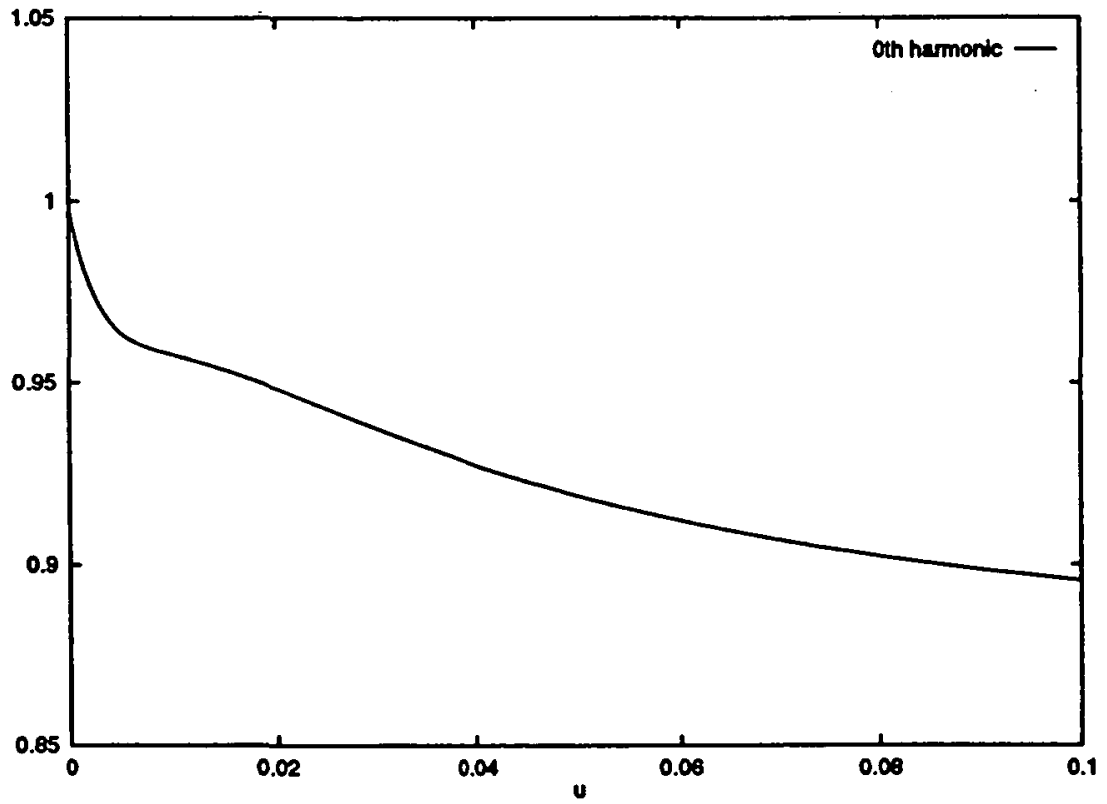

FIGURE 3. Mode $l=0$ tends to 0.88 as $u \rightarrow \infty$ 


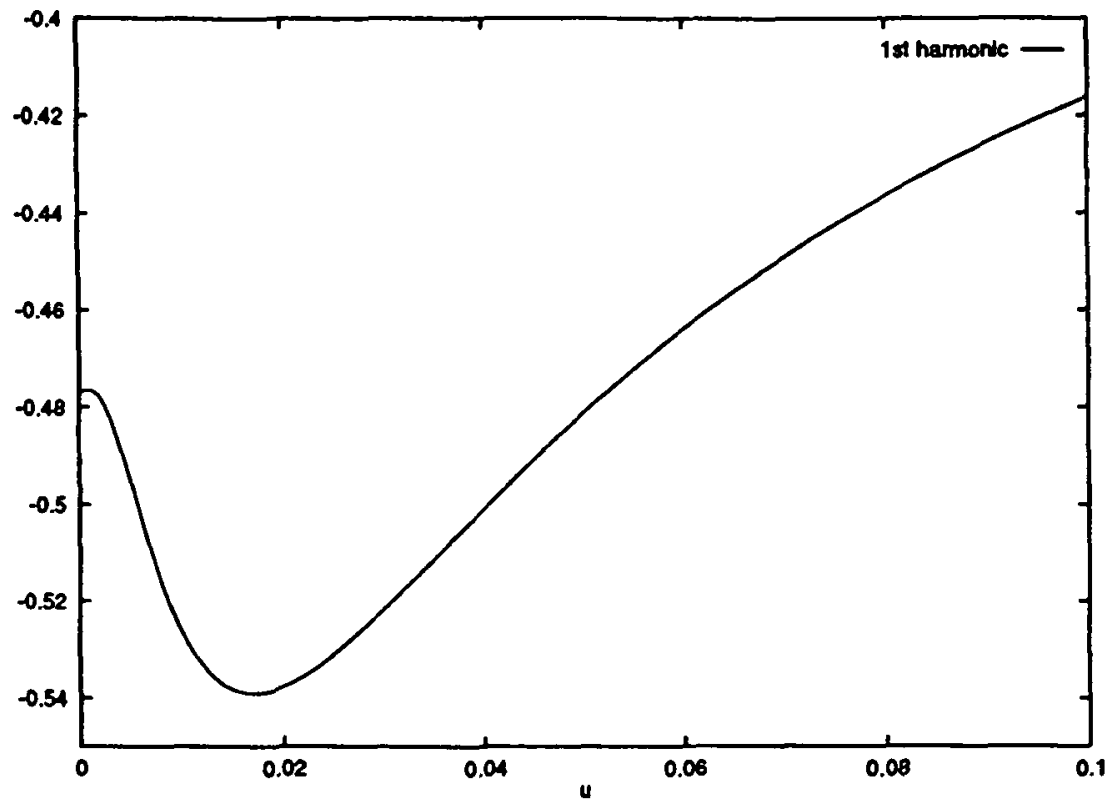

FIGURE 4. Mode $l=1$ tends to -0.35 as $u \rightarrow \infty$

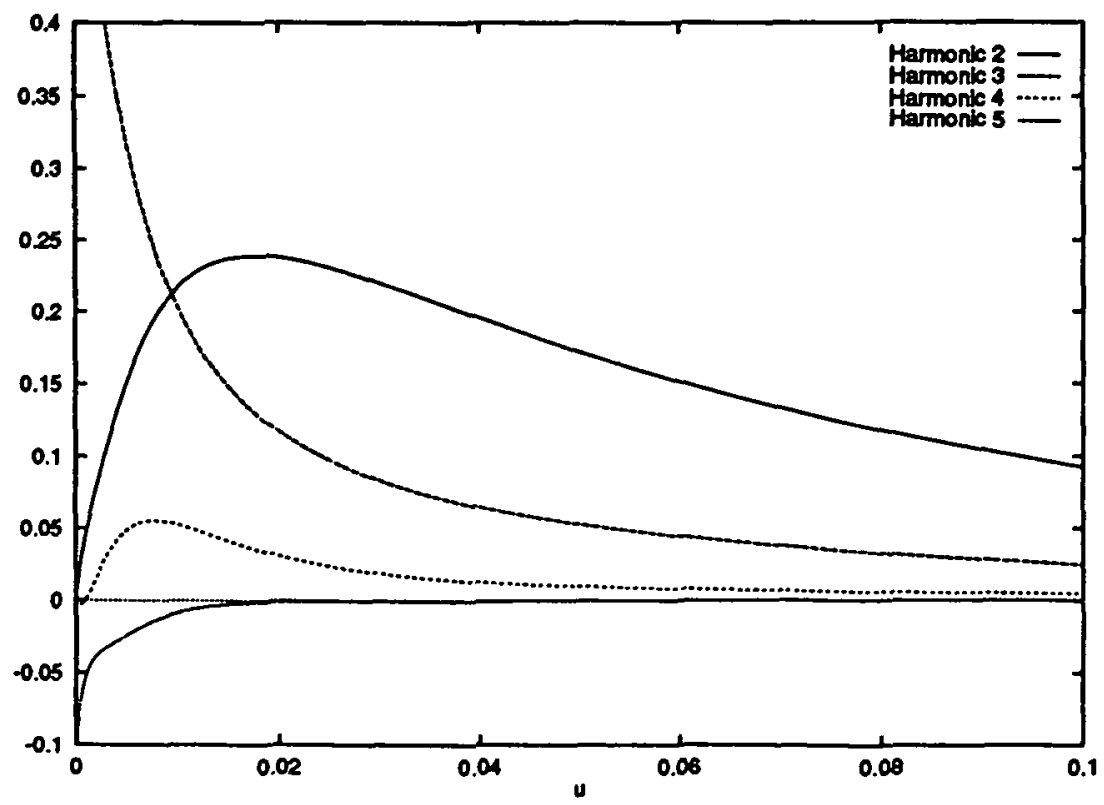

FIGURE 5. Interaction and decay of higher order modes 
parabolic problem, no maximum principle is evident.

Equation (2a) asserts that Bondi mass is a monotonically decreasing quantity. Figure 2 gives the decay of the numerical analogue of the Bondi mass. During the same period the surface area, which is conserved according to (2b), remains constant to within $2.6 \times 10^{-7}$. At large retarded time $(u=1.87)$ the surface area has still only varied very slightly $\left(3.5 \times 10^{-7}\right)$ from its initial value.

Further insights can be obtained by examining the evolution of the individual harmonics. The coefficients of the modes $l=0$ and $l=1$, which settle down to constant values at large retarded time, are shown in Figures 3 and 4 respectively. Figure 5 illustrates the type of evolution typical of higher order modes. Modes higher than $l=5$ are not shown - they decay at still faster rates.

Notice that mode two (the quadrupole moment), rapidly increases and then decays more slowly than the higher order modes. This behaviour appears to be a general feature of the evolution, independent of initial conditions. In each instance there is a rapid 'burst' of interaction between the modes, with mode two increasing significantly, followed by a period of decay. Mode zero experiences a kink while mode one has a local maximum and then a local minimum in the initial phase, followed (for both modes) by a later period of smooth monotonicity.

At a later retarded time of $u=1.9$, modes two and above have orders of $10^{-10}$ and smaller; the Bondi mass and the $l=0$ and $l=1$ harmonics have levelled out to constant values: we have reached the steady-state - the Schwarzschild solution.

In the linearized RT equation the modes are decoupled, so there is no interaction, modes zero and one are constant and the higher order modes decay independently.

Numerical integration took 35 seconds and 215 adaptive time-steps on a DEC $5000 / 125$ workstation, using a relatively unsophisticated linear systems solver.

\section{Other numerical schemes}

5.1. Standard finite differences In the absence of analytic and asymptotic solutions to the ART equation with which to compare our evolutions, it is extremely desirable to have a second numerical scheme to provide some degree of confirmation of results. For this purpose we have implemented a scheme based on standard finite differences.

It is feasible to perform all operations in a uniform pointwise basis. This approach generates banded rather than full matrices, making solution of the resulting linear systems much quicker than those which arise from the spectral method. However, the results obtained are less accurate, as centred differencing is not possible at the poles. In practice it is necessary to choose $n$ comparatively large, completely eroding the gains in efficiency made from the banded structure. Additionally, there is no natural interpolant. Should higher order interpolation be based on $z$ or $\theta$ ? 
5.2. Finite differences on an icosahedral grid Both the approaches discussed thus far should readily generalise to handle the full ' $2+1$ ' RT equation. We have partially implemented a third approach, based on a fine triangulation of the sphere [4]. Starting with an icosahedron, we perform an arbitrary number of barycentric subdivisions to obtain a fine, nearly uniform, mesh. By means of integral identities it is possible to derive finite difference expressions for the Laplacian and to thereby construct a numerical scheme. The book-keeping involved in such a grid is significantly more complicated than for the other approaches, but like the spectral transform method, this method does not suffer from special problems at the poles. To date we have only implemented an explicit time-stepping scheme, which is (not surprisingly) unstable except for very small $\Delta u$. An implicit version, although technically possible, would be more difficult to implement and would yield matrices with a very complicated (non-banded) structure.

\section{Conclusion and acknowledgement}

The Spectral Transform Method is well-suited to the integration of the ART equation. The degree of agreement with finite difference methods varies with the initial conditions, but is generally good. It is likely that discrepancies are attributable to the poor quality of finite difference approximations near the poles. Both approaches should readily generalise to the case of the full ' $2+1$ ' RT equation.

The natural decomposition into modes afforded by our approach is useful in interpreting numerical experiments. The most striking feature observed to date is the behaviour of the $l=2$ mode.

Further work is required to prove the convergence and stability of these methods, to give physical meaning to the initial conditions and to tie the numerical results in with the more conventional ' $3+1$ ' picture of spacetime.

We would like to thank Dr Leo C. Brewin for his advice and encouragement.

\section{References}

[1] G. L. Browning, J. J. Hack and P. N. Swarztrauber, "A comparison of three numerical methods for solving differential equations on the sphere", Mon. Wea. Rev. 117 (1989) 1058-1075.

[2] P. T. Chruściel, "Semi-global existence and convergence of solutions of the Robinson-Trautman (2-dimensional Calabi) equation", Commun. Math. Phys. 137 (1991) 289-313.

[3] B. Lukács, Z. Perjes, J. Porter and A. Sebastyen, "Lyapunov functional approach to radiative metrics", Gen. Relativ. Gravit. 16 (1984) 691-701.

[4] D. A. Prager, "Discretization of the two-sphere", Honours Thesis, Monash University, Melbourne, 1992. 
[5] I. Robinson and A. Trautman, "Some spherical gravitational waves in general relativity", Proc. Royal Soc. Lond. A262 (1962) 463-473.

[6] D. Singleton, "Robinson-Trautman solution of Einstein's equations", Ph. D. Thesis, Monash University, Melbourne, 1990. 